\title{
Cigarette smoke is a risk factor for severity and treatment outcome in patients with culture- positive tuberculosis
}

This article was published in the following Dove Press journal:

Therapeutics and Clinical Risk Management

6 October 2015

Number of times this article has been viewed

\author{
Hsiao-Chi Chuang ${ }^{1,2, *}$ \\ Chien-Ling Su ${ }^{1,2, *}$ \\ Hui-Chiao Liu ${ }^{2}$ \\ Po-Hao Feng ${ }^{1,3}$ \\ Kang-Yun Lee ${ }^{1,3}$ \\ Kai-Jen Chuang ${ }^{4,5}$ \\ Chun-Nin Lee L,2 $^{1,2}$ \\ Mauo-Ying Bien ${ }^{2,6}$ \\ 'Division of Pulmonary Medicine, \\ Department of Internal Medicine, \\ Shuang Ho Hospital, Taipei Medical \\ University, New Taipei, ${ }^{2}$ School of \\ Respiratory Therapy, ${ }^{3}$ Department \\ of Internal Medicine, ${ }^{4}$ Department \\ of Public Health, School of Medicine, \\ College of Medicine, ${ }^{5}$ School of Public \\ Health, College of Public Health and \\ Nutrition, ${ }^{6}$ Division of Pulmonary \\ Medicine, Department of Internal \\ Medicine, Taipei Medical University \\ Hospital, Taipei, Taiwan \\ *These authors contributed equally \\ to this work
}

Objective: Smoking has been associated with tuberculosis (TB); however, the effects of smoking on the effectiveness of TB treatment remain unclear.

Materials and methods: Data were retrieved from case notes and interviews of subjects registered in the TB-reporting system from 2010 to 2012. Study cases were defined as subjects with TB-positive sputum cultures, whereas the controls were defined as subjects with non-TBrelated pulmonary diseases. Statistical analyses included logistic regression and multivariate Cox proportional hazard regression models.

Results: A total of 245 cases with cultures positive for TB and 114 controls with non-TB-related pulmonary diseases and negative sputum cultures were recruited. Current smokers had the highest failure rate (33\%) for TB treatment, and they had the most severe pulmonary lesions based on chest X-ray grading. Current smokers had a 1.36-fold (95\% confidence interval 1.03-2.36, $P<0.05$ ) higher odds ratio for cultures positive for TB compared with nonsmokers. In subjects with TB-positive cultures, current smoking was associated with an increase in treatment days required for cultures to convert from positive to negative (hazard ratio $1.12,95 \%$ confidence interval 1.03-1.39; $P<0.05)$.

Conclusion: Longer periods of treatment may be required for TB patients who are current smokers.

Keywords: Mycobacterium tuberculosis, cigarette, immunity, chest X-ray

\section{Introduction}

Tuberculosis (TB) is a communicable disease, widespread throughout the world. The World Health Organization (WHO) estimated that there were 8.6 million TB cases, 1.3 million TB deaths, and an estimated 450,000 people who had developed multidrugresistant TB in 2013. ${ }^{1}$ With regard to treatment outcomes, the WHO reported that the success rate of treating TB was $87 \%$ among all new TB cases in 2011 . In Taiwan, TB has accounted for the highest incidence and mortality rate among all communicable diseases for many years. In 2012, for example, there were 12,338 TB cases and 626 TB-related deaths in Taiwan. Although the annual case numbers and incidence rate of TB in Taiwan have been decreasing, the success rate of treating TB is still below the world average reported by the WHO. ${ }^{1}$

The numbers of smokers have been continuously increasing in both developing and developed countries during the past several decades, and smoking has been associated with numerous diseases. For example, increasing evidence shows an association between smoking and TB. The correlation suggests that smoking is an important risk factor for the development of TB. Additionally, the risk of mortality from TB is higher in smokers. The possible mechanisms underlying these effects on TB infection
School of Respiratory Therapy, College of Medicine, Taipei Medical University, 250 Wuxing Street, Taipei II 0, Taiwan Tel +88622736 I66I ext 3516

Fax +88622739 I। 43

Email mybien@tmu.edu.tw 
in response to cigarette smoke include 1) dysfunctional mucociliary clearance, 2) decreased alveolar macrophage activity, 3) immunosuppression of the pulmonary lymphocytes, 4) inactive natural killer cells, and 5) dysfunctional pulmonary dendritic cells. Previous studies have shown that the severity of pulmonary lesions in $\mathrm{TB}^{2,3}$ and the TBrecurrence rate $^{2}$ are related to smoking. Therefore, smoking cessation represents an important factor in controlling TB epidemics. ${ }^{4}$ The objective of the present study was to investigate possible differences between TB-culture conversion ( $\geq 180$ days) in current smokers compared to nonsmokers and former smokers.

\section{Materials and methods Study population}

This investigation was a retrospective case-control study of cases reported between January 1, 2010 and December 31, 2012. The data were taken from 511 cases in the registry of the TB-reporting system in Taipei Medical University Shuang Ho Hospital in New Taipei (Taiwan). We included patients with TB-positive sputum cultures, of both sexes, aged $\geq 16$ years, whose treatment began during the period of study. An age-matched clinically selected control population was recruited from patients who had visited the chest clinic but who had been diagnosed with conditions other than TB. Subjects for whom the mandatory report was incomplete and for whom the missing data could not be retrieved were excluded. The diagnosis of TB was conducted using sputum cultures according to diagnostic standards. ${ }^{5}$ Sputum samples from the control subjects were also collected and cultured with negative results. The diagnosis of $\mathrm{TB}$ was reviewed at the beginning and end of the TB treatment, and cases for which TB was ruled out by the attending physician were excluded. The duration of sputum-culture conversion from positive to negative was calculated in days. The treatment procedure was the same for all the TB-positive subjects. Personal, clinical, and epidemiological data were obtained from the patients' clinical progress records. The microbiological laboratory data and medical records of the patients were reviewed.

Patients who smoked at the time of TB diagnosis were considered smokers ( $\geq 3$ months' current smoking). Subjects who had quit smoking at least 3 months prior to diagnosis were classified as former smokers, and those who had never smoked were classified as nonsmokers. This study was approved by the Taipei Medical University Joint Institutional Review Board. All patient records were anonymized and deidentified prior to analysis. The methods were carried out in accordance with the approved guidelines. All subjects received written and oral information prior to inclusion and provided informed consent.

\section{Grading of chest X-rays}

To quantify the severity of lesions observed in chest X-ray (CXR) images of the TB patients, a scoring method was applied to the present study. ${ }^{6}$ Standard full-size posteroanterior CXRs were obtained at the time of TB diagnosis. Briefly, the presence of nodules (1-2 mm or $>2 \mathrm{~mm}$ ), patchy or confluent consolidation, cavitation ( $\mathrm{mm}$ ), bronchial lesions or fibrosis, effusion, and lymphadenopathy were recorded in each lung (upper, mid-, or lower zones). Additionally, pulmonary effusion, lymphadenopathy, and the total percentage of each lung affected by any pathology was estimated. To grade the percentage of the lungs that were affected, a visual estimation of the extent of opacification, cavitation, or other pathology as a percentage of the visible lung was conducted. Dense and patchy opacification of a zone was graded. Dense opacification of a zone was graded as $100 \%$, whereas patchy opacification within a zone resulted in scores $<100 \%$ depending on the extent of opacification. The severity of TB CXRs was blindly scored by two chest physicians (KYL and PHF).

\section{Statistical analysis}

A logistic regression model was used to assess the associated risk of smoking in subjects with TB-positive cultures compared with that in subjects with TB-negative cultures. This model produced estimates of the odds ratios and the corresponding 95\% confidence intervals (CIs). Multivariate Cox proportional hazard regression was used to assess the hazard ratios and the corresponding 95\% CIs for those with positive sputum cultures relative to those with negative cultures. For comparisons among multiple values, one-way analysis of variance with Dunnett's post hoc test was used. All statistical analyses for this study were performed using SPSS 15.0 software (SPSS Inc, Chicago, IL, USA). The significance criterion was set at $P<0.05$.

\section{Results}

There were 511 subjects included in the registry during the period of study from 2010 to 2012; 245 TB cases eligible for inclusion in the present study were recorded. In this study, we enrolled a ratio of control-group (other pulmonary disease; culture-negative) to TB-group (culture-positive) individuals of $1: 2$. The demographic characteristics of subjects with TB-negative $(n=114)$ and TB-positive cultures $(n=245)$ are presented in Table 1. The percentage of smokers was similar $(27 \%)$ between the case and control groups, whereas the 
Table I Demographic characteristics of subjects with negative $(n=|| 4)$ and positive $(n=245)$ cultures from 2010 to 2012

\begin{tabular}{|c|c|c|}
\hline & $\begin{array}{l}\text { Negative cultures, } \\
\text { n (\%) }\end{array}$ & $\begin{array}{l}\text { Positive cultures, } \\
\text { n (\%) }\end{array}$ \\
\hline \multicolumn{3}{|l|}{ Sex } \\
\hline Female & $39(34)$ & $70(29)$ \\
\hline Male & $75(66)$ & $175(7 \mid)$ \\
\hline \multicolumn{3}{|l|}{ Age, years } \\
\hline Mean \pm SD & $57 \pm 18$ & $59 \pm 21$ \\
\hline Range & $20-96$ & $16-94$ \\
\hline \multicolumn{3}{|l|}{ BMI, $\mathrm{kg} / \mathrm{m}^{2}$} \\
\hline Mean \pm SD & $22 \pm 4$ & $22 \pm 4$ \\
\hline Range & $13-40$ & $12-33$ \\
\hline \multicolumn{3}{|l|}{ Marital status ${ }^{a}$} \\
\hline Unmarried & $16(14)$ & $42(17)$ \\
\hline Married & $98(86)$ & $203(83)$ \\
\hline \multicolumn{3}{|l|}{ Smoking status } \\
\hline Nonsmokers & $66(58)$ & $146(60)$ \\
\hline Current smokers & $17(15)$ & $32(13)$ \\
\hline Former smokers & $31(27)$ & $67(27)$ \\
\hline \multicolumn{3}{|l|}{ Alcohol consumption } \\
\hline No & $78(68)$ & $179(73)$ \\
\hline Yes & $36(32)$ & $66(27)$ \\
\hline \multicolumn{3}{|l|}{ Education } \\
\hline Less than high school & $61(53)$ & $129(53)$ \\
\hline High school or equivalent & $27(24)$ & $77(31)$ \\
\hline More than high school & $26(23)$ & $39(16)$ \\
\hline \multicolumn{3}{|l|}{ Socioeconomic status } \\
\hline High & $3(2)$ & $2(1)$ \\
\hline Middle & $93(82)$ & $204(83)$ \\
\hline Low & $18(16)$ & $38(16)$ \\
\hline \multicolumn{3}{|l|}{ HIV infection } \\
\hline Yes & 0 & 0 \\
\hline No & $114(100)$ & $245(100)$ \\
\hline \multicolumn{3}{|l|}{ Pulmonary cavity } \\
\hline Yes & 0 & $63(26)$ \\
\hline No & $114(100)$ & $182(74)$ \\
\hline \multicolumn{3}{|l|}{ CXR grading } \\
\hline Mean \pm SD & - & $33 \pm 29$ \\
\hline Range & - & $3-140$ \\
\hline \multicolumn{3}{|c|}{ Sputum positive to negative, days } \\
\hline Mean \pm SD & - & $242 \pm 108$ \\
\hline Range & - & $32-773$ \\
\hline \multicolumn{3}{|l|}{ Treatment outcome } \\
\hline Alive & - & $212(87)$ \\
\hline Dead & - & $33(13)$ \\
\hline
\end{tabular}

Note: -, data not available.

Abbreviations: SD, standard deviation; BMI, body mass index; CXR, chest X-ray.

nonsmokers accounted for $58 \%$ of the group with TB-negative cultures and $60 \%$ of the group with TB-positive cultures. Clinical and diagnostic characteristics indicated that no HIV coinfections were present at the time of diagnosis, and $26 \%$ presented with pulmonary cavitary lesions on CXR. The CXR grade of the group with TB-positive cultures was 33 . The number of days for positive cultures to become negative in the TB subjects was recorded: the average was 242 days (median 225 days) and the range was 32-773 days.
Figure 1A shows the treatment outcomes of patients with TB-positive cultures. The success rates of nonsmokers, former smokers, and current smokers were $77 \%, 78 \%$, and $67 \%$, respectively. The current smokers had the highest failure rate $(33 \%)$ of TB treatment. We then further investigated the median days of culture conversion in the nonsmokers, former smokers, and current smokers, which were 239, 275, and 272 days, respectively. Figure 1B illustrates the CXR grades of the nonsmokers, former smokers, and current smokers among the TB subjects. Within this group, current smokers had significantly higher CXR grades than nonsmokers $(P<0.05)$. No difference was observed between nonsmokers and former smokers.

Table 2 shows that the subjects with TB-positive cultures who were current smokers had an odds ratio of $1.36(95 \% \mathrm{CI}$ 1.03-2.36, $P<0.05$ ) relative to nonsmokers, after adjusting for age, sex, and body mass index. TB subjects who were former smokers had an odds ratio of 1.00 (95\% CI 0.71-1.89, $P=0.343$ ) relative to never-smokers. We further investigated the association between smoking and days for the sputum cultures to convert from positive to negative, as shown in Table 3. In subjects with TB-positive cultures, we observed an association between being a current smoker and an increased risk of a longer duration of culture conversion (hazard ratio $1.12,95 \%$ CI $1.03-1.39 ; P<0.05)$. There was no association observed between nonsmokers and former smokers.

\section{Discussion}

In this study, an association between current smoking and TB was revealed. These results were consistent with other studies. ${ }^{2,7-9}$ TB patients who were current smokers had a higher proportion of treatment failure (33\%) and higher severity of pulmonary lesions based on scores of the CXR-grading process. These findings may be related to a smoking-related alteration in the immunity of the TB patients, suggesting the possibility that smoking is a risk factor for adverse TB outcomes. Importantly, we showed that TB patients who were current smokers had a longer period of sputum-culture conversion.

In accordance with the WHO DOTS (Directly Observed Treatment, Short-course) program, ${ }^{10}$ Taiwanese policies target an $85 \%$ success rate of TB treatment. ${ }^{2}$ In the Centers for Disease Control report for Taiwan in 2012, ${ }^{3}$ new cases with TB-positive sputum cultures accounted for $36 \%$ of all new cases. Testing the mucus from the lungs in TB patients provides the best and most accurate diagnosis for active TB. To confirm our hypothesis that cigarette smoke influences the number of treatment days required for sputum cultures to 
A

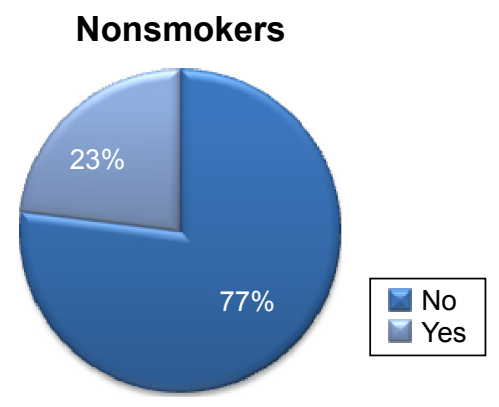

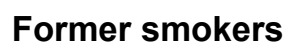

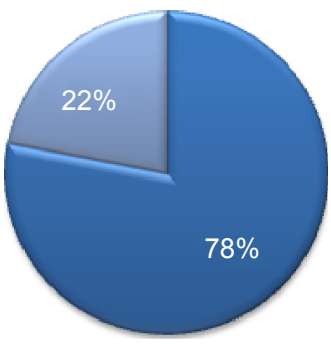

\section{Current smokers}

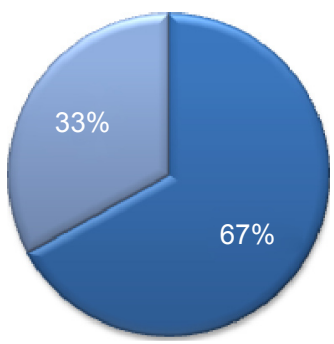

B

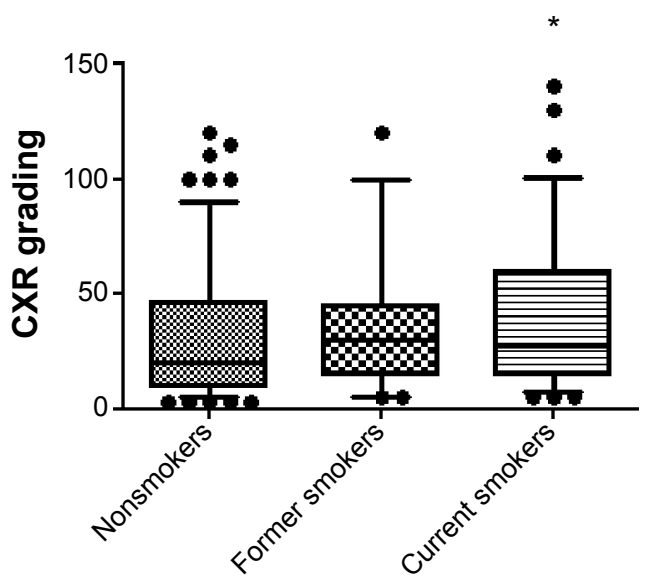

Figure I Smoking and the success rate of treating tuberculosis and CXR grading.

Notes: (A) Proportion of success rate of treating tuberculosis (TB) in patients with positive cultures who were nonsmokers, former smokers, and current smokers (No, treatment failure; Yes, treatment success). (B) Chest $\mathrm{X}$-ray (CXR) grading of patients with TB-positive cultures who were nonsmokers, former smokers, and current smokers. $* P<0.05$.

convert from positive to negative, subjects with TB-positive sputum cultures were therefore recruited for the study group. Within this group, the success rates of TB treatment were $77 \%$ in never-smokers and $78 \%$ in former smokers, whereas the rate was only $67 \%$ in current smokers. These treatmentsuccess rates of TB cases with positive sputum cultures were similar to the $66 \%$ recorded in 2010 in Taiwan after 12 months of follow-up. ${ }^{3}$ Both our results and the 2010 Taiwan report demonstrated average outcomes below the WHO target rate. The difference could be partially explained by TB-related risk factors, such as smoking. In the present study, there was a strong association between having TB-positive culture and being a current smoker. This association has also been

Table 2 Estimated ORs $(95 \% \mathrm{Cl})$ of the risk for tuberculosis patients with positive cultures among former smokers and current smokers

\begin{tabular}{ll}
\hline Smoking status & OR $(95 \% \mathrm{CI})^{\mathrm{a}}$ \\
\hline Nonsmokers & $\mathrm{I}$ \\
Former smokers & $\mathrm{I} .00(0.7 \mathrm{I}-\mathrm{I} .89)$ \\
Current smokers & $\mathrm{I} .36(\mathrm{I} .03-2.36)^{*}$ \\
\hline
\end{tabular}

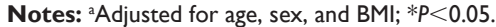

Abbreviations: ORs, odds ratios; $\mathrm{Cl}$, confidence interval; $\mathrm{BMI}$, body mass index. previously reported. ${ }^{2,7-9}$ Furthermore, smoking has been related to an increased risk of recurrence after successful anti-TB treatment. ${ }^{2}$ Yen et al showed that subjects with a current smoking rate of more than ten cigarettes/day had twice the risk of never- and former smokers. ${ }^{2}$ There was no difference between nonsmokers and former smokers among subjects with TB-positive cultures, which is consistent with the previous report by Leung et al. ${ }^{8}$ However, the effects of smoking on TB-culture conversion remain unclear.

Smoking has been reported to be harmful to human lungs, and to increase mortality and morbidity. ${ }^{11}$ We observed that the pulmonary lesions in current-smoker TB subjects were more severe, as measured by such criteria as treatment

Table 3 Hazard ratios of sputum-culture conversion duration (days) in subjects with tuberculosis-positive cultures

\begin{tabular}{ll}
\hline Smoking status & $\begin{array}{l}\text { Sputum-culture conversion, hazard ratio } \\
(\mathbf{9 5 \%} \text { confidence interval) }\end{array}$ \\
\hline Never-smokers & $\mathrm{I}$ \\
Former smokers & $0.83(0.58-1.17)$ \\
Current smokers & $1.12(1.03-1.39)^{*}$ \\
\hline
\end{tabular}

Notes: aAjusted for age, sex and body mass index; ${ }^{*} P<0.05$. 
outcome and CXR grading. Cigarette consumption is likely to increase the risk of mortality and morbidity in both high- and low-income countries. For example, the risk of TB death is approximately 4.5 times higher in smokers than nonsmokers, ${ }^{12}$ and smokers account for $61 \%$ of TB mortalities. ${ }^{12}$ Additionally, smoking was a factor associated with treatment failure and was a predictive factor of death. ${ }^{13}$ Notably, we observed an approximately 1.5-fold higher failure rate of TB treatment in current smokers compared to nonsmokers and former smokers. Treatment failure was mainly due to death during the treatment period. If survival is associated with smoking in TB patients, the pulmonary pathology may be more severe in smokers. In the present study, CXR grades in current smokers were significantly higher than in nonsmokers, suggesting that smoking caused the increased severity of TB lesions in the lungs. This concept is supported by previous work. ${ }^{7,11}$ Together, these results indicate that TB treatment-success rates, survival, and lung lesions are influenced by cigarette smoking. All the evidence implies that smoking may be associated with the efficacy of TB treatment.

If smoking causes faster development of TB lesions, then smoking may alter disease progress in TB patients. No difference has been observed in the time of diagnosis between smokers and nonsmokers with TB. ${ }^{7}$ A previous report indicated that in 287 new subjects with symptomatic TB, smoking was not identified as a predictive factor in any of the components of diagnostic delay. ${ }^{14}$ The time between the appearance of TB symptoms and the initiation of anti-TB treatment was similar in smokers and nonsmokers, but smokers presented with more severe lesions. Strikingly, we observed an association between current smoking and the number of days required for sputum-culture conversion. This finding suggests that smoking may prolong the required days in TB patients with positive cultures. The effects of smoking on disease progress have also been investigated in other diseases. For example, some of the chemical compounds in cigarette smoke, such as polycyclic aromatic hydrocarbons, can induce key drug-metabolizing enzymes, including cytochrome P450 and isoforms of the glucuronyltransferase families, ${ }^{15}$ which can modulate drug pharmacokinetics and efficacy in lung cancer treatment. Laki et al suggested that the identification of patients' smoking history is important for medical treatments. ${ }^{16}$ Based on our findings, we suggest that smoking-cessation activities should be included in TB care.

Immunopathological associations between smoking and TB have been revealed. ${ }^{17,18}$ Smoking decreases the defenses of the respiratory system, alters the mucociliary apparatus through cell destruction and dysfunction, exerts ciliostatic and ciliotoxic effects, and reduces lysozyme A activity. ${ }^{7}$ Cigarette smoke has been shown to cause structural changes in experimental mycobacterial strains. ${ }^{19}$ The fluid-producing functions of the lungs in both normal and TB patients have been shown to be increased by smoking. ${ }^{20}$ Smoking also induces alterations in both natural and acquired cell immunity that affect macrophages and leukocytes.

The limitations of the present study were that the recorded smoking history did not contain sufficient details to allow calculation of the number of cigarette pack-years, and that other recognized risk factors, such as indoor air pollution, were not included. Side effects and multidrug-resistant TB, which were not investigated in this study, may have affected the study outcomes. Additionally, in future studies, the TB drug used should be recorded to permit assessment of the possible interactions between the therapeutic regimens and cigarette smoking.

\section{Conclusion}

In conclusion, smoking was significantly associated with the period required for the sputum cultures to convert from TB-positive to TB-negative. This finding has important implications for TB-treatment strategies and control policy, and may be associated with the TB treatment-success rate in Taiwan, which is lower than the DOTS-targeted strategy. Smoking may increase the difficulty of treating TB, as well as prolong the days for each drug cycle. Public awareness needs to be encouraged to counter the continued prevalence of smoking and of TB.

\section{Acknowledgment}

The authors wish to thank Mrs Chiao-Ju Fu and Ching-Ling Li for technical assistance in this research. This study was founded by the Ministry of Science and Technology of Taiwan (MOST103-2314-B-038-018 and 104-2621-M-038-002MY3) and the Shuang Ho Hospital (104-SHH-HCP-015).

\section{Disclosure}

The authors report no conflicts of interest in this work.

\section{References}

1. World Health Organization. Global Tuberculosis Report 2013. Geneva: WHO; 2013.

2. Yen YF, Yen MY, Lin YS, et al. Smoking increases risk of recurrence after successful anti-tuberculosis treatment: a population-based study. Int J Tuberc Lung Dis. 2014;18:492-498.

3. Centers for Disease Control, Taiwan. Taiwan Tuberculosis Control Report 2012. Taipei: CDC; 2012.

4. Underner M, Perriot J. [Smoking and tuberculosis]. Presse Med. 2012;41: 1171-1180. French. 
5. [No authors listed]. Diagnostic Standards and Classification of Tuberculosis in Adults and Children. This official statement of the American Thoracic Society and the Centers for Disease Control and Prevention was adopted by the ATS Board of Directors, July 1999. This statement was endorsed by the Council of the Infectious Disease Society of America, September 1999. Am J Respir Crit Care Med. 2000;161:1376-1395.

6. Ralph AP, Ardian M, Wiguna A, et al. A simple, valid, numerical score for grading chest X-ray severity in adult smear-positive pulmonary tuberculosis. Thorax. 2010;65:863-869.

7. Altet-Gômez MN, Alcaide J, Godoy P, Romero MA, Hernández del Rey I. Clinical and epidemiological aspects of smoking and tuberculosis: a study of 13,038 cases. Int J Tuberc Lung Dis. 2005; 9:430-436.

8. Leung CC, Yew WW, Chan CK, et al. Smoking and tuberculosis in Hong Kong. Int J Tuberc Lung Dis. 2003;7:980-986.

9. Leung CC, Li T, Lam TH, et al. Smoking and tuberculosis among the elderly in Hong Kong. Am J Respir Crit Care Med. 2004;170:1027-1033.

10. World Health Organization. Global Tuberculosis Control. Geneva: WHO; 2011.

11. Mieczkowski B, Ezzie ME. Update on obstructive sleep apnea and its relation to COPD. Int J Chron Obstruct Pulmon Dis. 2014;9: 349-362.

12. Gajalakshmi V, Peto R, Kanaka TS, Jha P. Smoking and mortality from tuberculosis and other diseases in India: retrospective study of 43,000 adult male deaths and 35,000 controls. Lancet. 2003;362:507-515.
13. Santha T, Garg R, Frieden TR, et al. Risk factors associated with default, failure and death among tuberculosis patients treated in a DOTS programme in Tiruvallur District, South India, 2000. Int J Tuberc Lung Dis. 2002;6:780-788.

14. Casal Román M. [Study of delays in diagnosing symptomatic pulmonary tuberculosis]. Arch Bronconeumol. 2004;40:50. Spanish.

15. O’Malley M, King AN, Conte M, Ellingrod VL, Ramnath N. Effects of cigarette smoking on metabolism and effectiveness of systemic therapy for lung cancer. J Thorac Oncol. 2014;9:917-926.

16. Laki S, Kalapos-Kovács B, Antal I, Klebovich I. [Importance of drug interactions with smoking in modern drug research]. Acta Pharm Hung. 2013;83:107-120. Hungarian.

17. Yach D. Partnering for better lung health: improving tobacco and tuberculosis control. Int J Tuberc Lung Dis. 2000;4:693-697.

18. Maurya V, Vijayan VK, Shah A. Smoking and tuberculosis: an association overlooked. Int J Tuberc Lung Dis. 2002;6:942-951.

19. Shprykov AS, Zhdanov VZ, Lazovskaia AL, Levchenko TN. [Tobacco smoke condensate-induced structural changes in mycobacteria tuberculosis]. Probl Tuberk. 2002:39-41. Russian.

20. Pikas OB. [Effect of smoking and alcohol drinking on the moistureexcreting lung function]. Lik Sprava. 2000:65-67. Ukrainian.
Therapeutics and Clinical Risk Management

\section{Publish your work in this journal}

Therapeutics and Clinical Risk Management is an international, peerreviewed journal of clinical therapeutics and risk management, focusing on concise rapid reporting of clinical studies in all therapeutic areas outcomes, safety, and programs for the effective, safe, and sustained use of medicines. This journal is indexed on PubMed Central, CAS,

\section{Dovepress}

EMBase, Scopus and the Elsevier Bibliographic databases. The manuscript management system is completely online and includes a very quick and fair peer-review system, which is all easy to use. Visit http://www.dovepress.com/testimonials.php to read real quotes from published authors. 